\title{
Les stratégies d'insertion des « jeunes turcs » de
} France

Ceren Öztürk

\section{(2) OpenEdition}

1 Journals

Édition électronique

URL : https://journals.openedition.org/remi/3361

DOI : 10.4000/remi.3361

ISSN : $1777-5418$

Éditeur

Université de Poitiers

\section{Édition imprimée}

Date de publication : 31 décembre 2006

Pagination : 179-201

ISBN : 978-2-911627-43-9

ISSN : 0765-0752

\section{Référence électronique}

Ceren Öztürk, «Les stratégies d'insertion des « jeunes turcs » de France », Revue européenne des migrations internationales [En ligne], vol. 22 - $n^{\circ} 3$ | 2006, mis en ligne le 31 décembre 2009, consulté le 16 avril 2022. URL : http://journals.openedition.org/remi/3361 ; DOI : https://doi.org/10.4000/remi. 3361

Ce document a été généré automatiquement le 16 avril 2022.

(c) Université de Poitiers 


\title{
Les stratégies d'insertion des « jeunes turcs» de France
}

\author{
Ceren Öztürk
}

«Figés dans leur désir de ne rien perdre de leur culture, les 400000 Turcs de France ne se sont pas tous adaptés au mode de vie moderne $»^{1}$. Ce verdict sans appel émis par José-Alain Fralon dans Le Monde daté du 16 octobre 2004 reflète une vulgate assez commune aux discours ordinaires, médiatiques et politiques mais aussi académiques français. Il est évocateur d'une figure sociale immuablement arriérée pour ne pas dire franchement inassimilable. Aussi débuterons-nous, en guise d'antidote au poison de l'amalgame, en présentant quelques personnes, bien réelles, que nous avons rencontrées.

\section{Quelques portraits de « jeunes turcs»}

2 Gonca est l'aînée d'une famille de quatre enfants. Elle est arrivée en France à l'âge de 11 ans accompagnée de sa mère, ses frères et sa sœur. La famille rejoignait le père parti 4 ans plutôt. Gonca a été scolarisée en Turquie, jusqu'à la fin de l'école primaire. À son arrivée en France, elle a intégré une classe d'adaptation pour les élèves nonfrancophones, puis elle a poursuivi ses études sans heurts jusqu'à la fin du collège. À la sortie du collège, Gonca souhaitait continuer dans l'enseignement général et entreprendre des études pour devenir journaliste. Deux événements vont entraver son projet. Le premier est l'orientation scolaire que ses professeurs lui imposent à la fin de la troisième. Malgré sa relative réussite scolaire, Gonca est orientée vers une seconde technologique (TSA : Technologie des Systèmes Automatisés). Par la suite, elle tentera de réintégrer l'enseignement général mais, dans cette filière technique où prédominent les disciplines scientifiques, il lui sera difficile d'atteindre la moyenne générale nécessaire à sa réorientation. Ses notes lui permettront tout juste de poursuivre ses études en section "Sciences et Technologies Tertiaires : comptabilité et gestion ». Le deuxième événement qui empêchera Gonca de poursuivre ses études à l'Université relève des conditions économiques de sa famille. Au moment où la jeune femme obtient 
son baccalauréat, son père, ancien ouvrier puis chef d'entreprise dans le secteur du textile, perd son emploi. La précarité dans laquelle se trouve sa famille l'oblige à reconsidérer son projet professionnel. Gonca se voit contrainte d'entreprendre des études courtes en BTS (Brevet de Technicien Supérieur) "Comptabilité et Gestion ». Au bout de deux ans, elle arrête ses études sans obtenir son diplôme et recherche un travail. Après plusieurs emplois dans différentes structures, elle est embauchée avec un contrat à durée indéterminée dans un cabinet de comptable. C'est au moment où sa situation professionnelle et les conditions économiques de sa famille se stabilisent que Gonca revient à son projet initial. Tout en poursuivant son activité professionnelle, elle entreprend des études d'histoire à l'Université.

Mehmet est né en France, il est le cadet d'une famille de cinq enfants. Son père ancien commerçant émigre en France dans les années 1970. Dès son arrivée, il ouvre son propre commerce, une petite épicerie. Suite à une faillite, son père rejoint les migrants turcs qui travaillent dans les usines de construction automobile. Il travaillera dans la même usine pendant trente ans, jusqu'à sa retraite. Dans cette famille, l'éducation des enfants est la priorité des parents. Les grands frères de Mehmet, sous le contrôle strict et soutenu du père, ont entrepris de brillantes études scientifiques. Mehmet a bénéficié de l'aide et du soutien de ses frères pendant toute sa scolarité et lui aussi a été un élève brillant. Ses professeurs ayant remarqué ses capacités intellectuelles lui ont fait prendre une année d'avance par rapport à ses camarades. En troisième, malgré sa réussite scolaire, ses professeurs ont voulu l'orienter en lycée professionnel. Ce ne sont pas ses notes mais son comportement quelque peu agité qui justifie cette décision. Et c'est l'intervention de ses grands frères qui lui a permis de poursuivre ses études dans l'enseignement général. Après avoir obtenu son baccalauréat scientifique, Mehmet s'est inscrit à l'Université. Comme son grand frère, il a entrepris des études en informatique. Après avoir obtenu son DEUG (Diplôme d'Études Universitaires Générales), il a acquis sa première expérience professionnelle dans l'entreprise de son frère aîné. Il a quitté cette entreprise pour travailler dans une prestigieuse société publique en tant qu'informaticien. C'est encore une fois le réseau familial qui lui a permis d'accéder à cet emploi. Fort de ses expériences professionnelles passées, Mehmet est aujourd'hui sur le point d'intégrer une entreprise publique luxembourgeoise.

Elham est arrivé en France à l'âge de quatre ans, avec sa mère, sa sœur et son frère aînés. La famille y a rejoint son père, ancien instituteur contraint pour des raisons politiques de quitter la Turquie quelques années auparavant. La famille n'a pas vécu les difficultés d'accès au travail et au logement qui caractérisent le début du parcours migratoire de la majorité des migrants. Grâce à son statut de chef religieux, le père d'Elham dispose d'un solide réseau de relations. La famille est soutenue par la communauté alévie et bénéficie de conditions d'installation avantageuses. Elham, sa sœur et son frère sont rapidement scolarisés. Pendant leur scolarité, les enfants ont le soutien et l'aide permanents de leur mère qui, elle aussi était enseignante en Turquie. Elham dans les premières années de sa scolarité est un élève médiocre. Comme la majorité de ses camarades de classe, il n'aime pas l'école, il souhaite apprendre un métier. Il veut devenir boulanger. Sa mère est terrifiée devant ses résultats scolaires. Aidée par ses deux autres enfants, elle tentera autant que faire se peut, de motiver son fils. Les résultats scolaires d'Elham s'amélioreront lorsque, après le divorce de ses parents, il est contraint d'aider sa mère dans le petit commerce familial. Tout en travaillant, les trois enfants réussiront leurs études avec succès. Sa sœur aînée est 
diplômée d'une prestigieuse école de commerce. Son frère aîné intègre l'École des Ponts et Chaussées. Enfin, Elham après avoir obtenu un baccalauréat scientifique, prépare les concours des grandes écoles et il sera admis à l'École Polytechnique. Aujourd'hui Elham est sur le point de terminer sa formation à l'École des Ponts et Chaussées et il veut fonder une entreprise dans le secteur du bâtiment, en association avec son grand frère. Ces deux jeunes hommes souhaitent y employer essentiellement des personnes originaires de Turquie.

5 Sevgi est arrivée en France à l'âge de onze ans. Elle est l'ânée d'une famille de quatre enfants. Le père de Sevgi est un réfugié politique. Cet ancien instituteur travaille aujourd'hui en tant qu'agent d'entretien dans un établissement public. La mère de Sevgi est ouvrière. Le début du parcours scolaire de Sevgi est semblable à celui de nombreux enfants de migrant arrivés à un âge relativement avancé. Elle apprend le français dans une classe spécialisée pour les élèves non-francophones avant d'intégrer le collège. Dès le début de sa scolarité, cette jeune fille a un projet professionnel. Elle veut devenir aide-soignante. Mais, après avoir été orientée par ses professeurs vers un BEP (Brevet d'Études Professionnelles) Comptabilité et Gestion, elle abandonne son projet initial et elle poursuit ses études dans la voie qu'on lui a imposée. Après avoir obtenu un baccalauréat STT, elle s'inscrit à la faculté d'Histoire. Le choix de poursuivre des études universitaires est guidé par son nouveau projet professionnel. Sevgi veut passer le concours de recrutement des professeurs des écoles. Dans sa démarche, cette jeune femme n'est soutenue que partiellement par sa famille. Son père l'incite à terminer ses études le plus tôt possible car la scolarité prolongée de Sevgi constitue une charge importante pour cette famille ouvrière. Mais, malgré la pression familiale, elle n'abandonnera pas son projet personnel. Pour financer ses études, elle occupera des emplois étudiants. Actuellement Sevgi prépare le concours de recrutement des professeurs des écoles.

6 Ces quelques portraits le montrent, les situations sociales vécues par les jeunes originaires de Turquie sont beaucoup plus diversifiées que la doxa ne le laisse croire. Ils illustrent la diversité de situations sociales, de comportements, de choix, d'aspirations et de stratégies que l'on peut saisir au sein d'une population pourtant la plus souvent définie comme socialement et culturellement homogène. Loin de mener une existence à la marge de la société, certains d'entre eux réussissent à monter dans l'échelle sociale tandis que d'autres partagent le sort d'une partie de la jeunesse française plongée dans une expérience de vie que certains auteurs appellent « la galère » (Dubet, 1987).

\section{Précisions méthodologiques}

7 Ces portraits, comme les résultats qui suivent, sont issus de deux enquêtes ${ }^{2}$. Le premier travail de terrain, effectué à Rennes, visait à comprendre les modalités d'insertion socioprofessionnelle des femmes originaires de Turquie et d'étudier les relations de travail dans lesquelles elles sont engagées. Dans ce cadre, 18 entretiens ont été conduits. La deuxième enquête, menée en région parisienne, portait exclusivement sur les stratégies d'insertion sociale et professionnelle des enfants de migrants originaires de Turquie nés et/ou socialisés en France : 17 personnes âgées de 16 à 35 ans ont été interrogées par entretien semi-directif.

8

Dans les deux études, l'observation participante a également été utilisée. À Paris, notre engagement, bénévole puis salarié, dans l'association ACORT ${ }^{3}$ (Assemblée Citoyenne des 
Originaires de Turquie) nous a placée pendant un an dans une position où il nous était possible d'observer les jeunes dans leur quotidien. Les événements, les discours et les comportements qui faisaient sens pour notre enquête et en particulier les représentations des jeunes quant à leur famille, leur place au sein de la société française, la vie professionnelle, les relations de travail, la (dé) cohabitation avec les parents ou leurs quartiers de résidence ont été recueillies par ce moyen.

Pour constituer nos échantillons ${ }^{4}$, nous avons retenu deux critères de sélection: la revendication ou l'imputation d'appartenance au groupe «turc » et la socialisation en France. Nous avons donc cherché à rencontrer des jeunes scolarisés en France, au moins à partir du collège, de sorte que l'école française prend une part importante dans leur socialisation. La nationalité n'a pas été considérée comme un critère pertinent dans la mesure où la manière dont les jeunes s'identifient et sont catégorisés ne dépend qu'occasionnellement du critère juridique de nationalité. Nous n'avons pas non plus cherché à reproduire les subdivisions internes aux populations originaires de Turquie selon les appartenances confessionnelles, ethniques ou selon l'origine régionale. Cependant, nous avons essayé de solliciter des jeunes issus des sous-catégories ethniques, confessionnelles, politiques ou régionales, afin de rendre compte de l'hétérogénéité interne de ce groupe et nous avons cherché à intégrer le poids des caractéristiques "indigènes» dans la diversité des processus de construction identitaire des "jeunes turcs » et dans le déroulement de leurs trajectoires sociales. Parmi les enquêtés, nous comptons donc des personnes de nationalité française ou turque, des alévis et des sunnites, des Turcs et des Kurdes, des individus dont les parents proviennent d'un milieu rural et d'autres qui ont séjourné dans une grande ville avant d'émigrer en France. Les résultats que nous allons exposer n'ont donc aucune prétention à la représentativité statistique. Face à l'image stéréotypée du «jeune turc », c'est bien la variété de leurs stratégies d'insertion que nous voulons présenter.

\section{La stigmatisation des originaires de Turquie en France}

10 Après une quarantaine d'années de présence en France, les migrants originaires de Turquie et leurs descendants se sont vus attribuer une réputation peu enviable; ils seraient en "panne d'intégration ». Dès le début des années 1990, leur présence soulève des inquiétudes. Tribalat (1995) au terme d'une vaste enquête 5 réalisée sous sa direction, est la première à alerter les autorités et l'opinion publique sur le "défaut d'intégration " du groupe turc. Non contents d'être pauvres et peu éduqués, ils seraient porteurs d'une culture rurale fortement patriarcale, totalement incompatible avec celle de la société française.

11 Près de quinze ans se sont écoulés et les populations originaires de Turquie ne semblent pas pouvoir se défaire de cette réputation du migrant qui « pose problème ». La citation d'un journaliste du Monde qui ouvre cet article, témoigne de la pérennisation du stéréotype de l'incompatibilité supposée de leur mode de vie avec celui des Français. Cette représentation dévalorisante englobe toute personne identifiée comme "Turc ", y compris les jeunes nés et/ou socialisés en France dont les parents sont venus de Turquie. Désignés comme les représentants du groupe « turc », les jeunes originaires de Turquie sont sommés de répondre aux critères de l'intégration. En ce sens, leur 
trajectoire de socialisation devient un objet de scepticisme et la source d'incessantes tensions.

Les données de l'enquête MGIS (Mobilité Géographique et Insertion Sociale) relatives à la formation initiale, à la formation professionnelle et à la place des jeunes originaires de Turquie sur le marché du travail montrent une assez forte tendance à une reproduction à l'identique, qualifiée "d'immobilisme complet». Les "jeunes turcs» occuperaient les mêmes positions socioprofessionnelles que leurs parents et ce, dans les mêmes proportions : $80 \%$ seraient ouvriers et $10 \%$ travailleraient à leur compte. Ces jeunes continueraient, comme leurs parents, à faire appel à leurs réseaux de relations communautaires pour trouver un travail. Occupant des emplois dans les mêmes secteurs d'activités que ces derniers, la mobilité horizontale ne figurerait pas non plus dans les trajectoires socioprofessionnelles de ces deux générations. La cause de ce manque de mobilité inter-générationnelle serait à chercher, en premier lieu, dans les faibles performances scolaires des «jeunes turcs » qui, quant à elle, s'expliquerait par le désintérêt et les faibles aspirations de leurs parents vis-à-vis du fait scolaire (Tribalat, 1995).

13 Dans son explication des difficultés d'insertion des jeunes originaires de Turquie, Petek (1995) va encore plus loin: les «jeunes turcs» se caractériseraient par une situation de malaise et de confusion psychologique. La «communauté » et la «famille» en tant que lieu de « préservation des traditions villageoises anatoliennes » et « d'exacerbation de l'identité religieuse " constituent, selon Petek, l'obstacle majeur à l'émancipation des jeunes. Leurs difficultés scolaires sont analysées à partir de cette même logique qui consiste à opposer la culture familiale et la culture de la société d'accueil véhiculée par l'école. Ces auteurs expliquent donc la position désavantageuse qu'occupent les "jeunes turcs» dans la société française par le faible "degré d'intégration» de leurs parents. La «mauvaise intégration» des parents étant la cause des difficultés d'insertion des jeunes et leurs difficultés d'insertion venant confirmer la «mauvaise intégration » des populations originaires de Turquie. Ce raisonnement circulaire qui assigne une place prédéterminée aux enfants de migrants pourrait donc aisément s'appliquer aux générations à venir.

Loin d'éclairer la réalité sociale vécue par les familles migrantes et leurs enfants, ces discours académiques et médiatiques construisent et entretiennent la représentation dévalorisante du groupe turc et participent ainsi de la fabrication d'un stéréotype dépréciatif. Mais ces représentations ne sont pas sans effets. Elles interviennent dans les relations sociales en tant qu'outils de classement et de hiérarchisation des groupes ethniques en présence et participent ainsi à la construction de la frontière ethnique entre majoritaires et minoritaires, entre les « eux » et les « nous » (De Rudder, Poiret et Vourc'h, 2000). Cette stigmatisation contribue à l'infériorisation sociale et politique du groupe visé, à sa mise à l'écart ou en retrait des espaces de compétition (Réa et Tripier, 2003). Autrement dit, ces représentations dépréciatives pèsent sur les possibilités d'accès aux ressources économiques, sociales, éducatives et culturelles des populations originaires de Turquie dans la société française.

15 L'insertion sociale des « jeunes turcs » est ainsi évoquée sur le mode de la dénonciation de leurs difficultés, mais elle est rarement analysée dans ses processus constitutifs. C'est donc une approche déterministe, privilégiant la thèse de la reproduction des conditions de vie et de travail des immigrés, qui domine les discours sur la condition et le devenir des jeunes. Le registre culturel et le registre social sont, tour à tour ou 
conjointement, mobilisés pour expliquer "leurs difficultés » d'insertion sociale et professionnelle.

Loin de nier l'importance de l'appartenance sociale ni celle de l'appartenance culturelle dans les parcours individuels, il est nécessaire de les appréhender comme des dimensions toujours disposées à être négociées, retravaillées, rediscutées dans le cadre de multiples interactions. Il s'agit donc de prendre en compte le parcours social des jeunes dans sa totalité et de chercher à identifier les stratégies élaborées à partir de différentes sphères de socialisation. Cette déconstruction de l'homogénéité supposée de la catégorie "jeunes turcs » vise à restituer la diversité de leur situation, de leurs intérêts et de leurs identifications. En outre, cette démarche permet de replacer les relations ethniques dans l'ensemble des rapports sociaux (de classe, de genre, de génération...) et ainsi de relativiser le poids de la qualification culturelle et celui de la qualification sociale dans les trajectoires individuelles (Poiret, 2005).

Nous allons donc analyser les dynamiques observées dans l'univers familial, dans l'espace scolaire et sur le marché du travail. Faisant nôtre l'affirmation de Bastenier (2004) selon laquelle "les jeunes, même tenus dans une position minoritaire, demeurent des acteurs élaborant des stratégies vis-à-vis d'un monde qui s'impose mais sur lequel ils tentent d'avoir de l'influence en même temps qu'ils y entrent ", nous exposerons les différentes stratégies d'insertion sociale et professionnelle mises en place par les « jeunes turcs ».

\section{Ressources et contraintes dans l'univers familial}

18 La relative homogénéité des positions socioprofessionnelles occupées par les migrants originaires de Turquie en France combinée aux stéréotypes sur leur origine rurale masque les disparités en termes de capital économique, symbolique, éducatif et relationnel entre les familles de migrants. L'analyse des parcours familiaux dans le pays d'origine et dans la société d'installation est de ce point de vue intéressante. Elle révèle la diversité des trajectoires sociales des familles et partant la diversité des ressources dont elles sont porteuses (Santelli, 2001).

\section{Les ressources avant l'émigration}

Les histoires familiales antérieures à l'immigration interviennent dans les trajectoires d'insertion des jeunes originaires de Turquie à partir de deux logiques distinctes (Santelli, 2001). D'une part, elles procurent des ressources symboliques mobilisables dans le processus de construction identitaire. Le décalage entre les représentations dominantes concernant l'origine sociale des migrants et ce que les "jeunes turcs " perçoivent de leur histoire familiale, constitue un facteur qui peut permettre de se distancier de l'identité prescrite dévalorisante et de s'auto-valoriser. D'autre part, elles fournissent des ressources matérielles et relationnelles mobilisables qui ont une certaine incidence sur les conditions d'inclusion des familles dans le pays d'immigration et, partant, sur les parcours sociaux des enfants.

Le passage qui suit provient d'un entretien réalisé avec un jeune homme arrivé en France à l'âge de neuf ans. Ce court extrait révèle une forme de mobilisation des ressources symboliques de l'histoire familiale antérieure à l'émigration.

«On avait un destin sans doute très prometteur en Turquie, justement on avait la

chance d'avoir un grand-père très riche, il faisait partie de l'une des plus vieilles 
familles de Çine ${ }^{6}$. Le père de mon grand-père était un "bey"7 quoi. "

«Et en France c'était les années dures qui commençaient et il - le père - a vraiment travaillé dur. [...] Je me souviens de lui quand il partait vendre des trucs sur la Côte d'Azur, des choses importées de Turquie, comme les tapis des choses comme ça. On a vendu aux Halles des jouets, y en avait un qui guettait pour voir si les flics venaient. On a vécu pas mal de choses comme ça. Ce n'était pas la misère mais c'était la bohême quoi. » (Yalım, architecte)

21 Son père, un commerçant fortuné qui voulait consolider sa position sociale en devenant maire de sa ville natale, a dû fuir son pays suite au coup d'État militaire de 1980. À son arrivée en France, la trajectoire professionnelle du père se caractérise par la précarité et l'instabilité. Il est vendeur ambulant. Mais quelques années suffisent à cet homme d'affaire confirmé pour remonter dans l'échelle sociale. Grâce aux transferts d'argent effectués par sa famille depuis la Turquie, il ouvre un petit commerce. En s'appuyant sur les ressources communautaires, il développe différentes activités commerciales et reconstitue de cette manière une partie du capital économique perdu lors de l'émigration. Cependant, dans le discours de Yalım, plus que cette ascension rapide réalisée en France, c'est le parcours familial dans le pays d'origine qui fait l'objet d'une identification positive. Il est probable que l'histoire familiale lui procure des ressources symboliques et économiques plus riches que le seul statut socioprofessionnel occupé par son père en France. Aussi Yalım mobilise-t-il les ressources familiales antérieures à l'émigration dans l'évaluation qu'il fait des conditions économiques d'existence de sa famille lors de la période d'installation en France : il qualifie de " bohême » la situation de précarité dans laquelle se trouve sa famille.

Les phénomènes de transmission sur trois générations ne sont pas seulement des ressources symboliques mobilisées dans le processus de construction identitaire. Ils peuvent avoir un impact important sur les parcours familiaux dans le pays d'arrivée. Posséder une certaine somme d'argent ou disposer d'un réseau de relations large peut faciliter considérablement l'installation et fournir un contexte plus favorable à l'enfant. La trajectoire professionnelle du père de Yalım est à ce titre révélatrice. Au-delà des caractéristiques personnelles du père - qui soulignent par ailleurs l'importance des dispositions sociales et professionnelles antérieures à l'émigration - c'est l'utilisation des ressources économiques provenant de Turquie qui explique sa réussite professionnelle. Cette ascension sociale rapide dans le pays d'émigration, et les ressources économiques qui en découlent, ont des incidences positives sur le parcours social de ses enfants et en particulier sur leur parcours scolaire. Par exemple, contrairement à d'autres enfants de migrants qui ont vécu les premières années de leur scolarité dans la solitude, Yalım a pu bénéficier lors de ses études de la présence et du soutien constants de sa mère. Les revenus du père étant suffisamment élevés, sa mère a pu se permettre de se consacrer à la scolarité et à l'éducation de ses trois enfants. La réussite scolaire de Yalım qui lui a permis d'entreprendre des études dans une prestigieuse école d'arts appliqués parisienne a probablement été favorisée par les conditions familiales dont il a bénéficiées.

\section{Ressources et contraintes familiales en France}

Les immigrés originaires de Turquie, qu'ils soient paysans, ouvriers, commerçants ou artisans, riches ou pauvres, scolarisés ou analphabètes, migrants politiques ou économiques, et quelles que soient les raisons qui ont motivé leur émigration, ont eu 
accès à une portion très limitée du marché de l'emploi français. Beaucoup d'entre eux ont débuté leur carrière en tant qu'ouvrier et le sont restés tout au long de leur parcours professionnel. Les positions professionnelles des pères et l'inactivité - supposée ou réelle - des mères ont maintes fois été invoquées pour expliquer les difficultés d'insertion des «jeunes turcs ». Cependant, les récits des enquêtés nous conduisent à reconsidérer la relation entre les parcours sociaux des deux générations et à prendre en compte d'autres éléments que les seules carrières professionnelles dans l'évaluation des positions sociales familiales.

En effet, comme les parcours familiaux antérieurs à l'émigration, les trajectoires sociales des familles dans le pays d'installation fournissent aux jeunes d'importantes ressources. Les responsabilités politiques, syndicales, religieuses assumées par les parents dans la société d'installation participent à la construction du capital symbolique et relationnel des familles dans le pays d'immigration. Ils constituent pour les «jeunes turcs» des ressources relationnelles importantes en même temps qu'une source d'identification positive.

"Mon père, quand il est venu en France, il a ouvert un commerce, ça a foiré et après il est entré à l'usine. À l'usine rapidement il est devenu syndicaliste, il a appris le français. Il parle bien le français mon père. Et il a travaillé essentiellement avec ce syndicat. Il a été quand même actif, il a ouvert le "dernek" ${ }^{8}$ près de chez mes parents, "dernek" qui regroupe actuellement 700 familles... Mon père était un grand homme. Même si c'était un petit homme, c'était un grand homme quand même. Il était respecté parce que c'était un des seuls qui parlaient français, donc les gens venaient régulièrement à la maison, mon père s'occupait de leurs papiers, il les aidait. » (Mehmet, 26 ans, informaticien).

Le point d'aboutissement du parcours migratoire du père de Mehmet ressemble à celui de milliers de migrants originaires de Turquie du début des années soixante-dix. Après avoir été ouvrier pendant 30 ans, dans une usine automobile, il est aujourd'hui à la retraite. Malgré cette position socioprofessionnelle peu avantageuse, Mehmet bénéficie tout au long de son parcours, d'un réseau relationnel et d'une reconnaissance importante grâce aux différentes responsabilités assumées par son père - son engagement associatif et syndical, le soutien qu'il apporte aux membres de son groupe d'appartenance.

La reconnaissance sociale dont bénéficient les parents - et les pères en particulier au sein de leur groupe d'appartenance, joue un autre rôle dans la trajectoire sociale des jeunes. Qu'il s'agisse d'un statut existant avant l'émigration ou d'une reconnaissance acquise à travers les rôles et statuts extra professionnels, cela procure aux familles - et donc à leurs enfants - un capital relationnel important qui facilite l'accès des jeunes à certaines ressources matérielles et humaines relevant du milieu "communautaire». Les emprunts d'argent qui se font sur la base de la confiance et de l'honneur sont fréquents dans le groupe des originaires de Turquie. Nombreux sont les jeunes qui ont bénéficié de l'aide financière de leur entourage proche lorsqu'ils ont débuté leur carrière d'entrepreneur ou qui ont acquis une première expérience professionnelle dans les entreprises des co-ethniques. 


\section{L'interdépendance entre les itinéraires individuels des membres d'une même famille}

En étudiant l'intégration urbaine des familles ouvrières à Turin, Gribaudi (1987) note que «dans une situation sociale où les ressources et les possibilités individuelles se modifient en fonction des charges familiales, les conditions économiques familiales constituent un élément important qui intervient dans les trajectoires socioprofessionnelles des individus». L'étude des parcours sociaux des «jeunes turques» révèle, elle aussi, l'interdépendance entre les trajectoires individuelles des membres d'une même famille. L'histoire de Gonca, le premier de nos portraits, permet de montrer l'impact des dynamiques intra-familiales dans l'élaboration des stratégies d'insertion professionnelle.

Lorsqu'elle était encore collégienne, Gonca voulait devenir journaliste. Mais ce projet se heurte à une orientation scolaire plus subie que choisie ${ }^{9}$ tandis que son père perd son emploi au début des années 1990. Elle est donc obligée de reconsidérer son projet professionnel.

«C'est dur, quand tu sais que tes parents sont au chômage et qu'il faut payer quand même le loyer. Mon frère et moi, on n'a pas pu aller à l'université à cause de ça. [...] Mes parents ne le savent pas, je ne leur ai pas dit, mais c'est pour ça que je n'ai pas suivi des études longues. Actuellement je paye le loyer, les factures et la mutuelle de mes parents, les charges fixes quoi. Mon frère lui, il paye les courses. » (Gonca, 25 ans, comptable).

Pour Gonca, la limitation de son ambition professionnelle à une situation d'employée est liée à la précarité dans laquelle se trouve sa famille plus qu'à une absence d'aspiration à la mobilité. Cette jeune comptable envisage d'ailleurs de reprendre ses études.

Par ailleurs, la mise au travail des aînés allégeant les charges familiales et procurant des ressources supplémentaires, les cadets semblent bénéficier des conditions matérielles plus favorables pour une insertion sociale réussie. L'entrée dans la vie active de Gonca, et dans une moindre mesure, celle de son frère ${ }^{10}$, influencent de manière positive le parcours professionnel de leurs cadets qui bénéficient d'un contexte économique familial plus favorable à la poursuite d'études ${ }^{11}$. Ainsi ce qui semble déterminant dans la distribution des ressources n'est pas tant la taille des familles que les liens de solidarité et d'obligation qui se créent entre ses membres.

\section{Cohabitation avec les parents}

Dans une situation d'insuffisance matérielle, les jeunes développent d'autres stratégies pour mobiliser les ressources familiales. Pour une partie des parents originaires de Turquie, la décohabitation des enfants, tant pour les filles que pour les garçons, doit s'inscrire dans une démarche de vie maritale ayant obtenu leur assentiment et non dans une démarche d'autonomie individuelle. Certains jeunes élaborent leurs projets en manipulant cette norme. L'assignation à résidence dans la maison paternelle jusqu'au mariage et/ou l'obligation d'hospitalité des parents leur confère la possibilité de développer des stratégies d'insertion socioprofessionnelle complexe sans interrogation pressante sur leur avenir (Quiminal, Timera, Fall, Diarra et Diouf, 1997).

«J'avais le choix entre reprendre mes études et me prendre un appartement. Je travaillais à mi-temps alors je ne pouvais pas faire les deux en même temps. À 
l'époque, j'ai choisi les études. » (Zeynep, 31 ans, assistante juridique et étudiante en Faculté de Droit).

32 Aînée des enfants, Zeynep est l'un des personnages clef de sa famille. C'est elle qui surveille la scolarité de ses jeunes frères et sœurs. C'est elle qui joue le rôle d'interprète lorsque les parents doivent affronter les institutions officielles. Enfin, c'est elle qui apporte un soutien financier à la famille lorsque les parents sont dans l'impossibilité de faire face aux dépenses quotidiennes. Ces responsabilités constituent un obstacle insurmontable pour Zeynep qui, malgré une autonomie financière acquise et des aspirations à une autonomie totale, ne prend pas le risque de passer à l'acte. Pourtant, les responsabilités assumées dans la sphère familiale, n'expliquent pas à elles seules le choix de Zeynep. En effet, du fait de son statut de femme, l'assignation à résidence dans le domicile des parents pèse plus intensément sur elle. Même lorsque ses frères et sœurs arrivent à un âge où ils peuvent prendre le relais dans l'aide apportée aux parents, Zeynep repousse son départ et elle ne se décide à franchir le pas que dix ans après sa première tentative de décohabitation. Paradoxalement, cette contrainte a une influence positive sur son parcours socioprofessionnel. Elle lui permet de reprendre ses études et d'accéder ainsi à un niveau de qualification plus élevé. Par la suite, elle peut se lancer dans une carrière professionnelle, découvrir et développer plus librement des intérêts et des relations sociales pouvant se transformer en de nouvelles et importantes ressources.

Le choix de Zeynep met en scène la complexité des paramètres qui entrent en ligne de compte dans la prise d'une décision qui apparaît, de prime abord, fort simple. Son parcours montre les possibilités de manipulation des contraintes et des ressources qui relèvent de différentes sphères de socialisation. Son choix résulte en effet de la prise en compte de nombreux paramètres comme ses aspirations professionnelles et scolaires, les conditions économiques de sa famille ou les contraintes qui pèsent sur elle dans la sphère familiale en tant que fille aînée.

Ces différents exemples montrent combien est variable la manière qu'ont les «jeunes turcs " de se référer à leur milieu familial et d'utiliser les ressources symboliques, relationnelles et matérielles qu'ils y trouvent pour élaborer des stratégies. Leurs choix et leurs comportements différent dans leurs déterminations et dans leurs résultats, en fonction des conditions matérielles propres à chaque famille ainsi qu'en rapport aux rôles sociaux - selon le genre et selon le rang dans la fratrie - qu'ils assument. Cependant, les modes d'insertion socioprofessionnelle des jeunes ne relèvent pas uniquement de l'univers familial et l'analyse doit s'étendre à des milieux de référence extérieurs en englobant les parcours scolaires des « jeunes turcs ».

\section{Les parcours scolaires}

L'accès au marché du travail étant fortement déterminé par l'acquisition de diplômes valorisés et reconnus, une scolarisation "réussie » peut être garante d'une insertion socioprofessionnelle sans heurt tandis que le manque de diplôme peut être synonyme de disqualification sociale. La certification scolaire n'est pas seulement un minimum requis pour l'insertion professionnelle, elle constitue plus globalement un gage de conformité social qui a des répercussions dans tous les domaines de la vie. C'est ce qui explique la vivacité des débats relatifs au destin scolaire des enfants de migrant. Considéré comme l'un des vecteurs principaux de la socialisation et de la mobilité 
sociale, l'espace scolaire est investi d'attentes particulières aussi bien du côté des familles immigrées et de la société dominante que par les "jeunes turcs». Or les difficultés scolaires de nombreux enfants de migrants originaires de Turquie sont bien réelles. Elles se caractérisent par un taux de redoublement élevé, de nombreuses orientations en classe spécialisée, des cursus scolaires dans les filières techniques courtes. Pour expliquer ce désavantage scolaire, la recherche a souvent mis en avant les facteurs culturels et sociaux, focalisant ainsi l'analyse sur le milieu familial. Pour notre part, nous proposons une analyse des parcours scolaires centrée d'une part sur l'élève acteur de son insertion scolaire et d'autre part sur les processus d'ethnicisation des rapports sociaux à l'œuvre dans l'univers de l'école.

\section{Famille et École}

\section{L'impact relatif des ressources éducatives familiales}

Des familles immigrées qui disposent d'un capital culturel élevé ne parviennent pas toujours à influencer de manière positive les parcours scolaires de leurs enfants. En créant des conditions défavorables à la transmission des ressources éducatives, une attitude parentale générale qui se caractérise par la distance relationnelle et le manque d'intérêt vis-à-vis de la scolarité de l'enfant influe de manière négative sur les trajectoires scolaires. À l'autre extrémité, c'est-à-dire dans les familles où le capital culturel fait défaut, les enfants ne semblent pas être voués à un destin scolaire inexorablement défavorable. Le contrôle strict et soutenu de la scolarité des enfants par les parents, qui peut aller jusqu'aux punitions corporelles, et leurs encouragements jouent un rôle positif. La mobilisation des aînés ou d'autres membres de la famille pour soutenir la scolarité des plus jeunes constitue une autre réponse à la situation de manque de ressources éducatives des parents.

39

Les propos d'un futur avocat sur les raisons de sa réussite scolaire résument une partie des éléments que nous venons d'évoquer. Ni sa mère, ni son père n'ont connu l'école. 
Cependant par leurs encouragements et par leur attention, ils ont su faire une place effective à l'enfant-écolier au sein de la configuration familiale.

«Par rapport à ma scolarité, quand j'étais en primaire j'étais assez réservé mais je travaillais. Mais ça vient du fait que mon père n'a pas étudié, ma mère non plus. Donc ils voulaient qu'on ait une éducation. [...] Mon père m'encourageait quand je recevais de bonnes notes. Il me donnait des bonbons, des choses comme ça. J'avais un frère qui était très studieux aussi, qui a aussi fait l'université. Donc en prenant comme exemple mon frère, j'étais obligé de faire aussi bien que lui. » (Soley, 27 ans, étudiant à l'Institut d'Études Judiciaires)

Le témoignage de Soley met également en lumière la dimension identitaire du rapport au savoir et rend compte des mécanismes d'identification complexes qui participent du rapport du jeune à la réussite scolaire ${ }^{13}$. Dans cette perspective, l'apport des frères et sœurs aînés ne se limite pas à une aide à l'apprentissage des connaissances. Les résultats scolaires des aînés, leur rapport à l'école et aux études constituent une référence qui peut déterminer, selon des modalités diverses, le déroulement de la scolarité des plus jeunes. En ce qui concerne Soley, dans la relation qu'il entretient avec son frère, la compétition et le mimétisme se conjuguent et influencent son positionnement vis-à-vis de l'école et de la réussite scolaire. Et cette logique compétitive et mimétique ne prend sens qu'en référence à sa volonté de répondre aux attentes des parents.

\section{Les aspirations des familles et leur investissement scolaire}

41 Dans l'explication des résultats scolaires des enfants de migrants, certains chercheurs ont tenté de prendre en compte le poids de l'origine nationale. Vallet (1996) adopte cette perspective et conclut que les élèves d'origine étrangère obtiennent de meilleurs résultats scolaires que leurs condisciples de même catégorie sociale. L'hypothèse retenue pour expliquer cet avantage est celle de la motivation particulière des familles immigrées qui, elle, se comprend à partir de facteurs comme les statuts socioprofessionnels inférieurs occupés par les parents dans la société d'installation, la volonté de promotion sociale qui anime ces familles et la scolarisation entendue comme le principal instrument de mobilité disponible dans le contexte migratoire. Toutefois, ce constat doit être relativisé. En établissant un lien direct, entre les aspirations familiales, l'investissement des familles dans la scolarité de leurs enfants et la réussite de ces derniers à l'école, cette démarche tend d'une part à homogénéiser les attentes et les pratiques des familles en matière éducative et, d'autre part, occulte le rôle joué par l'enfant dans son insertion dans le milieu scolaire et dans la construction de son rapport au savoir.

Pour notre part, les témoignages recueillis montrent que toutes les familles ne s'investissent ni de la même manière ni avec la même intensité dans la scolarité de leurs enfants. Pour certains parents, l'école n'est pas toujours l'ultime vecteur d'ascension sociale et, de ce fait, ne fait pas l'objet d'une grande mobilisation. Les raisons de ce désintérêt sont, selon nous, à rechercher dans la stratégie d'insertion sociale adoptée par les membres du groupe familial. Autrement dit c'est l'interdépendance entre les trajectoires individuelles qui doit faire l'objet d'analyse.

Le passage qui suit provient d'un entretien réalisé avec un jeune homme arrivé en France à l'âge de six mois. Kemal n'a pas bénéficié du soutien de sa famille - de ses parents pas plus que de ses deux frères aînés - lors de sa scolarité ni lors de son 
parcours professionnel. Au contraire le prolongement de ses études et les perspectives professionnelles qu'il lui offre ont été source de tensions entre les membres du groupe familial.

«Je ne comprends pas les familles turques, je pense que ça ne va jamais évoluer [leur position sociale en France] parce que, les familles ne veulent pas. Mon frère est fâché avec moi parce que je ne veux pas travailler avec lui dans son entreprise, dans le bâtiment. Il veut que je travaille avec lui parce qu'il a besoin de moi. Moi si j'ai des potentiels, si j'ai moyen de faire autre chose, pourquoi je ne ferai pas mieux qu'eux. Eux ils pensent toujours à rester dans la même chose, c'est la mentalité. » (Kemal, 25 ans, commercial).

Les groupes familiaux optent, en fonction des ressources dont ils disposent, pour différentes stratégies d'insertion. Les initiatives entrepreneuriales modestes construites sur la base familiale et qui nécessitent la mobilisation des énergies et des compétences des membres d'une même famille en sont un exemple. Dans ce cas précis, le prolongement de la scolarité des enfants peut signifier une perte de ressources humaines pouvant mettre en danger la stratégie collective d'insertion sociale. Les opportunités professionnelles qu'une éventuelle réussite scolaire rendrait accessibles, pourraient favoriser les stratégies d'insertion individuelles et, par conséquent, le détachement du jeune de son groupe familial en même temps que du projet collectif.

Guidés par cette même logique d'insertion collective, les choix scolaires et professionnels des «jeunes turcs" peuvent être orientés en fonction des besoins du groupe familial. L'attrait de certains jeunes pour les filières de comptabilité et de gestion s'explique sans doute par l'importance de l'activité indépendante dans le groupe des originaires de Turquie. Cette stratégie qui consiste donc à acquérir des compétences pouvant être réinvesties dans l'entreprise familiale permet d'avoir accès à un débouché sûr tout en contribuant à la consolidation ou à l'amélioration de la situation familiale.

\section{Des trajectoires scolaires marquées par l'appartenance ethnique et l'appartenance de classe}

La réussite ou l'échec des jeunes dans le système scolaire n'est pas seulement déterminé par leurs capacités personnelles et par les ressources matérielles et éducatives que propose leur univers familial. Le rôle de l'école dans l'orientation des choix et dans la définition des aspirations individuelles s'avère déterminant.

Qu'ils aient immigré en France ou qu'ils y soient nés, les « jeunes turcs » restent, pour la plupart, considérés comme porteur d'une différence indélébile. Dans leurs activités quotidiennes, ils découvrent l'identité dévalorisante qui leur est attribuée et, partant, la place inférieure qui leur est assignée au nom de leur appartenance ethnique. C'est dans ce contexte marqué par les rapports de domination entre majoritaires et minoritaires que les «jeunes turcs" sont amenés à construire une identité (Moreau, 1999). Dans ce processus, l'école joue un rôle important puisqu'elle organise l'entrée des enfants de migrant dans la société globale. Ils s'y heurtent aux normes dominantes du pays d'installation, inculquées par les enfants français du même âge, par les enseignants et autres représentants du groupe majoritaire et ils prennent conscience de la position d'altérité qui les caractérise ${ }^{14}$. Or, cette position n'est pas sans conséquence sur les stratégies de réussite scolaire que les jeunes élaborent. 
«La première année, je ne foutais absolument rien en classe à part chanter dans mon petit coin. [...] Après il y a eu comme un déclic. [...] Et tout d'un coup, je suis devenue la première de la classe, je voulais tout apprendre. [...] Comme si je devais compenser un manque; le vide là, il fallait le combler par quelque chose d'autre et je l'ai comblé par le savoir. [...] Après, tous les profs étaient fiers de moi. "On aimerait avoir 35 Yeliz dans notre classe", des remarques comme ça qui, d'ailleurs, ont fait que les élèves m'excluaient davantage. Par exemple, les profs n'arrêtaient pas de dire, mais contre leur gré finalement; "vous avez vu Yeliz, elle vient d'arriver, mais elle parle mieux que vous le français". Du coup les élèves me voyaient comme quelqu'un qui était le contraire d'eux, donc je les mettais mal à l'aise finalement. De ce point de vue-là j'ai toujours été exclue. [...] Même aujourd'hui, je suis à la fac, quand je fais une bonne remarque, quand je pose une bonne question... [silence] je vois bien le regard de mes camarades. C'est l'idée de dire: “Ce n'est pas normal qu'elle soit mieux que nous, parce que c'est une étrangère". [...] Pour eux, une occidentale est forcément plus intelligente que quelqu'un qui vient du tiers-monde. » (Yeliz, 23 ans, étudiante en philosophie)

Yeliz est arrivée en France à l'âge de sept ans. Son rapport aux études est en partie déterminé par sa perception des situations d'interactions scolaires. Ses propos sur ses premières années à l'école et sur la suite de son parcours révèlent l'importance des processus de différenciation - tels qu'ils sont ressentis par l'élève - dans la construction des carrières scolaires. Selon Goffman (1975 [1963]), l'individu stigmatisé peut réagir diversement à sa situation. L'une des possibilités qui s'offre à lui est de "chercher à améliorer sa condition en consacrant en privé beaucoup d'efforts à maîtriser certains domaines d'activité que, d'ordinaire, pour des raisons incidentes ou matérielles, on estime fermés aux personnes affligées de sa déficience ». C'est cette stratégie qu'adopte Yeliz et il en résulte un important investissement dans les études et une amélioration considérable de ses résultats scolaires.

$\mathrm{Au}$ sein de l'école, la problématique ethnique ne constitue pas le seul vecteur d'altérité qui s'impose à l'individu (Quiminal, Timera, Fall, Diarra et Diouf, 1997). Les positionnements des jeunes et les stratégies qui en découlent articulent en général la dimension de classe à celle de l'ethnicité. Le discours de Mehmet permet de montrer la manière dont les jeunes manipulent la définition exogène de l'identité fondée conjointement sur l'appartenance de classe et l'appartenance ethnique dans l'élaboration de leur stratégie de réussite scolaire.

«J'étais bon en maths et c'est toujours ça qui m'a porté. De toute façon, la littérature, je ne sais pas si ce sont mes frères qui me l'ont dit ou moi-même je l'ai compris, ce n'était pas pour nous. [...] Pour moi de toute façon c'était les maths. Et donc, oui, c'était par rapport à ça que j'ai été à la fac, c'est par rapport à ça que j'ai fait de l'informatique... [silence] tout, en fait. Je pense encore que ce n'est pas pour nous la littérature. En tout cas, dans le milieu social où j'étais, ce n'était pas pour nous. [...] Il y a beaucoup d'informaticien étranger. Je pense que les Orientaux ne sont pas mauvais pour la logique, je ne sais pas si je suis en train de dire quelque chose de raciste, mais ils captent très bien. Mes collègues français des fois, ils sont "out", ils ne comprennent rien. » (Mehmet, 26 ans, informaticien).

Dans la construction de son rapport aux études, Mehmet mobilise l'idée selon laquelle les enfants de migrants, surtout s'ils sont issus d'une famille ouvrière, auront moins de succès dans les filières littéraires. Se considérant comme peu doué dans les matières qui nécessitent une mobilisation importante de la langue, Mehmet oriente tout son parcours en fonction de l'aisance qu'il a dans les filières scientifiques. Aisance qui, selon lui, s'explique en rapport avec son appartenance ethnique. Ainsi, Mehmet reprend la différence qui lui a été imposée. Il s'affirme différent, mais attribue une 
valeur positive à cette différence. Comme le montre la suite de son témoignage, les catégories de pensée que ce jeune homme intègre depuis sa socialisation dans l'univers de l'école subsistent tout au long de son parcours social.

\section{Les parcours professionnels}

\section{Mobilisation des ressources ethniques et familiales dans l'accès à l'emploi salarié}

51 Les témoignages recueillis montrent une mobilisation fréquente des réseaux de relations familiales et ethniques ${ }^{15}$ dans l'accès à l'emploi mais aussi dans les étapes suivantes des parcours professionnels. Pourtant, cette utilisation des réseaux communautaires entre en contradiction avec le discours des "jeunes turcs». Ces derniers expriment souvent leur volonté de ne pas s'employer dans les structures appartenant aux co-ethniques pour différentes raisons. En premier lieu, c'est à cause des conditions de travail, particulièrement dures et précaires. Les niveaux de rémunération bas, le non-respect du droit du travail et le faible prestige social qui caractérisent les emplois du secteur "ethnique » ne créent guère d'attrait pour les jeunes. Deuxièmement, ce refus se comprend en référence aux normes sociales qui régissent les relations de travail. Pour certains jeunes, appartenir au même groupe ethnique ou à la même famille que son employeur représente un désavantage considérable puisque, alors, les règles qui régissent les relations de travail ne relèvent plus seulement $\mathrm{du}$ cadre réglementaire mais aussi des usages coutumiers. Les possibilités de contester des pratiques considérées comme illégales dépendent de la nature plus ou moins contraignante des relations sociales engagées sur le lieu de travail. Dans ces circonstances, face à un employeur appartenant au même groupe ethnique ou à la même famille, les jeunes estiment disposer d'un pouvoir de négociation moindre.

Malgré ces réticences, les jeunes construisent - et parfois sont contraints de construire - leur parcours d'insertion en s'appuyant sur leurs ressources ethniques et familiales. Cependant, les finalités visées par le recours au salariat dans les entreprises «turques» diffèrent en fonction du niveau de qualification du jeune. Pour certains, l'emploi dans ces structures constitue un tremplin qui permet d'accumuler de l'expérience pour pouvoir se diriger ensuite vers des secteurs plus valorisés. Pour d'autres, le recours à ces réseaux représente le moyen de se maintenir sur le marché du travail et de prétendre à un emploi au-dessus de ses qualifications.

Gölge est la seule enquêtée à avoir quitté le système scolaire sans obtenir de qualification reconnue. Néanmoins, en s'appuyant sur son réseau de relations, elle parvient à trouver un travail dans le cabinet de comptabilité d'un ami turc. Au début de son parcours professionnel, cet emploi lui permet de ne pas être confrontée à l'inactivité mais aussi d'acquérir des compétences par le biais d'une formation informelle qu'elle reçoit dans l'entreprise. Cependant, ces deux années d'expérience ne suffisent pas à lui assurer une insertion professionnelle "réussie». Le manque de certification restreint fortement les possibilités de cette jeune femme en termes d'emploi, l'obligeant à faire appel aux entreprises turques où les employeurs sont moins exigeants quant aux critères d'embauche. 


\section{Mobilisation des ressources ethniques et familiales dans la création d'entreprise} une stratégie de sortie de la précarité. Les "jeunes turcs» disposent de ressources auxquelles les membres d'un autre groupe ethnique - minoritaire ou majoritaire n'ont pas accès. Les réseaux de relations ethniques leur permettent de constituer une clientèle sûre et fidèle. L'initiative entrepreneuriale suivante illustre cette forme de mobilisation des ressources ethniques en rapport avec la nature de l'activité.

« [...] Bien après que j'ai intégré Arc-en-Ciel ${ }^{16}$, j'avais un rôle dans l'organisation des fêtes $^{17}$, donc je commençais à voir comment ça se passait bien. À l'époque je n'avais pas du tout l'idée de faire des fêtes ou quoi que ce soit. Un jour j'ai téléphoné à Sertan et je lui ai dit: "On fait une soirée“. J'ai contacté Emrah aussi, l'ancien DJ d'Arc-en-Ciel, et Bektaş aussi. Ça ne sert à rien d'avoir 10 personnes, si au bout du compte tu n'as que 2 personnes qui font les choses. Et les autres, bon, même s'ils apportent une aide matérielle et humaine, au bout du compte tu les tires plus que... En plus, il y a des risques financiers là-dedans, ce n'est pas l'association. [...] La première soirée qu'on a faite, on a eu plus de 400 personnes. Seconde soirée, on a eu plus de monde et on a vu que la mayonnaise avait prise, que les gens trouvaient ça sympa et maintenant ça fait 3 ans qu'on fait ça. » (Soley, 27 ans, étudiant à l'Institut d'Études Judiciaires).

Soley est connu parmi la population originaire de Turquie résidant en région parisienne. Il doit cette célébrité à sa fonction de présentateur de l'émission de radio ${ }^{18}$ préparée par les « jeunes turcs » membres de l'association ACORT. En mobilisant les ressources relevant de ce milieu associatif, Soley crée une petite structure commerciale. Il organise des soirées privées festives pour les jeunes. Son initiative 
s'appuie sur plusieurs ressources : les compétences acquises comme bénévole au sein de l'association; le réseau de relations qui s'est constitué ; et enfin, la fonction occupée dans l'émission de radio qui lui permet de faire la publicité de ses soirées et de bénéficier d'une certaine reconnaissance auprès de la clientèle-cible.

\section{Valorisation des liens avec le pays d'origine}

il a obtenu un diplôme d'informaticien de niveau BAC +2 . Lorsque nous l'avons rencontré, Derya était sans activité. Son projet consistait à suivre une formation de coiffure en France, puis de partir en Turquie pour y exercer ce métier. Derya s'était donc inscrit à une formation de coiffeur tout en continuant à chercher un emploi dans l'informatique. Quelques mois après notre entretien, il nous a annoncé qu'il venait d'être embauché dans une société d'informatique et avait abandonné son projet de retour en Turquie. Dans son cas, le retour en Turquie constitue une réponse provisoire à sa situation de précarité professionnelle. Mais pour d'autres jeunes, la valorisation des liens avec la Turquie fait partie d'une stratégie non de sortie de la précarité ou de la marginalité socioprofessionnelle mais d'ascension sociale.

« Il y a six ans, en Turquie il n'y avait rien, mais aujourd'hui il y a une explosion. Il y a des boîtes d'architectures éphémères ${ }^{19}$ qui se créent. Ça fuse de partout la créativité. C'est ça qui fait qu'aujourd'hui j'ai envie de me mettre à mon compte pour travailler avec la Turquie. Donc moi, d'une pierre deux coups, j'aimerais travailler pour ces boîtes turques, en faisant profiter de mon expérience à la française. Parce que la "French Touch" c'est un truc qui marche à travers le monde. » (Yalım, 32 ans, architecte)

61 Yalım a un parcours professionnel qu'il juge réussi. Diplômé d'une prestigieuse école d'arts appliqués parisienne - l'École Boulle -, il n'a pas eu de difficulté à s'insérer sur le marché du travail français. Dans un premier temps, il a été salarié d'une agence d'architecture pendant cinq ans. Après le dépôt de bilan de cette société, Yalım, l'expérience aidant, a rapidement trouvé un autre emploi dans ce même secteur. Malgré cette stabilité professionnelle, Yalım envisage de créer une activité indépendante où il pourrait mettre à profit ses compétences et acquis professionnels «à la française » et ses liens avec la Turquie.

\section{L'« autre » réseau ethnique}

Le recours aux réseaux ethniques ${ }^{20}$ peut aussi jouer dans le sens d'une restriction des possibilités d'embauche et participer à un cantonnement dans des secteurs d'activité dévalorisés. Face à cette situation, les «jeunes turcs » tentent de mettre en place des stratégies collectives qui réunissent les membres de leur groupe d'appartenance et en particulier d'autres "jeunes turcs" disposant d'un niveau de qualification élevé et d'une expérience sur le marché du travail. L'idée est de créer un site Internet regroupant les coordonnées des entreprises turques localisées en Turquie ainsi que des

Revue européenne des migrations internationales, vol. 22 - n³ | 2006 
entreprises françaises où certains jeunes ont déjà été employés ou ont effectué des stages.

En créant ce site, les jeunes de l'ACORT souhaitent formaliser les réseaux de relations existants et étendre leur portée en les mutualisant. Cette démarche se base d'une part, sur la valorisation des liens avec le pays d'origine, à travers le recours aux entreprises turques de Turquie et, d'autre part, sur le partage de leurs expériences professionnelles et relationnelles dans les entreprises françaises. Ils prévoient donc la constitution d'un fichier regroupant les sociétés «françaises » où, lors de leur passage, ils ont laissé une «bonne » impression en tant que " jeune turc ». Il s'agit en effet de mobiliser l'identité ethnique et de tenter de modifier son contenu aux yeux des personnes appartenant à la société majoritaire.

Le deuxième mobile de la création de ce site Internet est directement lié à une volonté de se soustraire aux discriminations à l'embauche. C'est pourquoi ils prévoient un fichier regroupant les coordonnées d'entreprises où ils estiment ne pas avoir rencontré de pratiques discriminatoires. Une liste noire des sociétés "discriminantes » est également envisagée. Certes, il n'est pas sûr que cette démarche soit efficace. L'évaluation que chaque individu fera des situations de rencontres avec les majoritaires dans le cadre des relations de travail est subjective et, de ce fait, ne peut pas être considérée comme une source d'information infaillible. Des comportements qui ne relèvent pas d'une logique discriminatoire peuvent être perçus comme tels et un traitement inégalitaire ayant un fondement ethnique peuvent être considéré comme « naturel » ou «normal » (De Rudder, Poiret et Vourc'h, 2000). Mais que cette initiative soit efficace ou non, elle représente une stratégie collective qui se donne pour objectif l'amélioration des conditions d'accès des jeunes au marché du travail en optimisant l'utilisation des ressources existantes au sein de groupe d'appartenance.

\section{En guise de conclusion}

65 L'étude des processus d'insertion sociale et professionnelle et les stratégies que cette approche permet de saisir, montre la diversité des situations sociales, des logiques d'actions, de comportements et de choix, des aspirations et des mobilisations des « jeunes turcs ». Nos observations sur le déroulement des parcours des jeunes ainsi que sur leur milieu familial n'ont donc pas de prétention à la généralité. Elles constituent la base d'une réflexion qui interroge les stéréotypes véhiculés sur cette population.

L'analyse des parcours sociaux dévoile les rapports sociaux inégalitaires dans lesquels les «jeunes turcs » sont engagés. Les sphères de socialisation désignées comme étant vecteur d'intégration, l'école par exemple, ne fonctionnent pas toujours de manière positive dans les parcours sociaux des enfants. De l'autre côté l'univers familial qui a souvent été désigné comme un obstacle à l'intégration peut être porteur de ressources considérables. Cette ambivalence met à mal les discours univoques sur l'intégration et, plus particulièrement, les approches qui mettent en avant la reproduction de la classe sociale et la reproduction de la «culture d'origine " comme cause des problèmes rencontrés par les jeunes dans leur insertion sociale et professionnelle. Ils montrent que chaque sphère de socialisation - famille, école, monde du travail - constituent des espaces où les jeunes négocient leur place en fonction des ressources dont ils disposent et des contraintes qu'ils rencontrent. 
67 L'injonction d'intégration adressée aux «jeunes turcs» sous-tend l'idée d'une distanciation par rapport à leur famille et plus largement par rapport à leur groupe d'appartenance. Or leurs stratégies d'insertion révèlent une logique inverse. Leur agir se comprend à partir des dynamiques observées dans les ensembles familiaux et non pas comme l'expression d'individualités isolées obéissant à la logique des grandes institutions de l'intégration. Tout en tentant de dépasser et/ou de modifier les frontières de leur appartenance, les «jeunes turcs» mobilisent leurs ressources propres et bénéficient d'une solidarité à laquelle ils tiennent. Cette ambivalence s'explique moins par «l'ardeur» de leur attachement au groupe d'appartenance que par leur position socioéconomique au sein de la société française.

Le vécu des individus ne peut pas être tenu pour le simple résultat des déterminismes sociaux ou des actions individuelles. Il se construit à travers les multiples processus de socialisation qui influent sur l'horizon des possibles de chaque individu. Dans la confrontation avec les différentes sphères de socialisation - dans la famille, à l'école ou dans le monde du travail -, l'ethnicité est un ressort significatif de l'action des « jeunes turcs ». Si la stigmatisation dont ils font l'objet contraint la manière dont ils se définissent eux-mêmes, ils parviennent aussi à manipuler cette identité dominée, tant individuellement que collectivement, pour donner naissance à des stratégies d'insertion variées.

\section{BIBLIOGRAPHIE}

BASTENIER Alain (2004) Qu'est-ce qu'une société ethnique ? Paris, PUF, 346 p.

CHARLOT Bernard (1997) Du rapport au savoir, Paris, Economica, $112 \mathrm{p}$.

DE RUDDER Véronique, POIRET Christian et VOURC'H François (2000) L'inégalité raciste, Paris, PUF, $224 \mathrm{p}$.

DUBET François (1987) La galère : jeunes en survie, Paris, Fayard, 471 p.

FRALON José-Alain (2004) Plus Turcs que Turcs, Le Monde, 16.10.2004.

GRIBAUDI Mauricio (1987) Itinéraires ouvriers, espaces et groupes sociaux à Turin au début du XXème siècle, Paris, Édition des Hautes Études en Sciences Sociales, 264 p.

IRTIS-DABBAGH Verda (2003) Les jeunes issus de l'immigration de Turquie en France, Paris, L'Harmattan, $427 \mathrm{p}$.

LAHIRE Bernard (1998) La réussite scolaire en milieux populaires ou les conditions d'une schizophrénie heureuse, Ville-École-Intégration, n 114, pp. 104-109.

LAHIRE Bernard (1996) Des réussites sociales multiformes en milieux populaires, MigrantsFormation, $\mathrm{n}^{\circ} 104$, pp. 22-27

PAYET Jean-Paul (2000) Violence à l'école et ethnicité. Les « raisons pratiques » d'un amalgame, VEI-Enjeux, L'universel républicain à l'épreuve. Discrimination, ethnicisation, ségrégation, $\mathrm{n}^{\circ} 121$, pp. 190-200. 
PETEK Gaye (1995) La course d'obstacle des jeunes issus de l'immigration turque, MigrantsFormation, $\mathrm{n}^{\circ} 101$, pp. 153-167.

POIRET Christian (2005) Articuler les rapports de sexe, de classe et inter-ethniques : quelques enseignements du débat nord-américain, Revue Européenne des Migrations Internationales, 21 (1), pp. 195-226.

QUIMINAL Catherine, TIMERA Mahamet, FALL Babacar, DIARRA Hamédy et DIOUF Babacar (1997) Les jeunes filles d'origine africaine en France : Parcours scolaires, accès au travail et destin social, Migrations Etudes, $\mathrm{n}^{\circ} 78,20 \mathrm{p}$.

REA Andrea et TRIPIER Maryse (2003) Sociologie de l'immigration, Paris, Éditions La Découverte, 122 p.

SANTELLI Emmanuelle (2001) La mobilité sociale dans l'immigration: Itinéraires de réussite des enfants d'origine algérienne, Presse Universitaires du Mirail, Toulouse, 302 p.

TIMERA Mahamet (1999) Logiques familiales, communautaires et scolarisation de jeunes filles d'origine africaine noire en France, Revue Formation, Emploi, Marseille (CEREQ), n 65, pp. 57-75.

TRIBALAT Michèle (1995) Faire France : Une enquête sur les immigrés et leurs enfants, Paris, La Découverte, $302 \mathrm{p}$.

VALLET Louis-André (1996) L'assimilation scolaire des enfants issus de l'immigration et son interprétation : un examen sur des données françaises, Revue française de pédagogie, $\mathrm{n}^{\circ} 117$, pp. 7-27.

\section{NOTES}

1. Fralon José-Alain, « Plus Turcs que Turcs », Le Monde, 16 octobre 2004.

2. Mémoires de maîtrise à l'Université Rennes 2 et de DEA à l'Université Paris 7, sous la direction de Christian Poiret.

3. ACORT est une association de loi 1901 soutenu financièrement par des organismes publics comme le FASILD ou le Conseil Régional. Elle se situe dans le $10^{\text {ème }}$ arrondissement de Paris où se concentre une partie importante de la population originaire de Turquie. Cette structure a été fondée en octobre 1981 sous le nom d'ATT (Association des Travailleurs Turcs), à la suite des grèves de la faim des travailleurs de la confection en 1980. Les initiatives de cette association se concentrent autour de préoccupations politiques, sociales et culturelles. La lutte contre les discriminations, le droit de vote des étrangers et les questions liées au code de la nationalité se comptent parmi les domaines d'action de cette structure. Les activités orientées vers les populations originaires de Turquie suivent les impositions des pouvoirs publics : c'est-à-dire œuvrer pour l'intégration de ces populations et jouer le rôle d'intermédiaire entre ces dernières et les autorités locales. Dans cette perspective, l'association aide les migrants dans leurs démarches administratives, leur proposent des cours de français, met en place des permanences d'accompagnement juridique, etc. Elle organise également des cours de soutien scolaire en direction des enfants et mène des activités de médiation entre les parents et les écoles du $10^{\text {ème }}$ arrondissement.

4. Nous utilisons ce terme sans prétention à une quelconque représentativité. L'essentiel, pour les besoins de notre enquête étant d'étudier, au moins partiellement, les variations du phénomène qui nous intéresse.

5. Enquête «Mobilité Géographique et Insertion Sociale ( (Institut National d'Études Démographiques) et l'INSEE (Institut National de la Statistique et des Études Économiques). 
6. Ville natale de l'enquêté.

7. Un bey est un titre turc désignant à l'origine un « chef coutumier » (lignage, clan, tribu...). Sous l'Empire ottoman, les bey ont pu être des princes vassaux ou des sortes de préfets de l'Empire dans les provinces éloignées. Aujourd'hui, c'est un titre de respect donné à une personne à qui l'on veut montrer de la déférence (grand propriétaire terrien, le sens donné ici, patron, supérieur hiérarchique...).

8. En turc dans le texte, en français : association.

9. Nous ne développerons pas ce point, cependant il faut préciser que dans les parcours scolaires des « jeunes turcs » l'orientation apparaît comme un problème récurrent.

10. Le frère de Gonca poursuit ses études en BTS et occupe un emploi à mi-temps.

11. Sa petite sœur vient de passer son baccalauréat $S$ et envisage d'entreprendre des études à l'université. Son frère cadet est étudiant en psychologie. Les études entreprises par ces deux jeunes supposent une scolarité longue et une mise au travail tardive par rapport à leurs aînés.

12. Parmi les 17 pères, 2 n'ont pas été scolarisés, 6 d'entre eux ont obtenu le certificat d'étude primaire, 2 autres ont poursuivi leurs études jusqu'à la fin du collège, 3 migrants ont obtenu leur diplôme de fin de lycée et enfin 4 autres ont poursuivi des études supérieures et ont atteint le niveau de $\mathrm{BAC}+2$ ou $\mathrm{BAC}+4$. Les mères ont en général un niveau d'études moins élevé que les pères. Parmi les 17 mères, une seule n'a pas été scolarisée, 10 ont terminé leurs études primaires, 2 ont été scolarisées jusqu'à la fin du collège, 3 autres ont obtenu leur diplôme de baccalauréat et une seule a poursuivi des études supérieures.

13. Selon Bernard Charlot (1997: 84) : « apprendre fait sens en référence à l'histoire du sujet, à ses attentes, à ses repères, à sa conception de la vie, à ses rapports aux autres, à l'image qu'il a de lui-même et à celle qu'il veut donner aux autres ».

14. Sur les processus de ségrégation interne et l'ethnicisation des rapports sociaux en milieu scolaire cf. PAYET Jean-Paul (2000).

15. Lors de l'enquête en région parisienne, parmi les 14 personnes de l'échantillon ayant eu une activité salariée, 7 ont fait appel à un moment donné de leur parcours professionnel, à des coethnique : 5 de ces 7 personnes ont travaillé dans une entreprise gérée par des co-ethniques ; les 2 autres ont bénéficié de soutien dans leur démarche de création d'entreprise sous forme d'aide financière. Enfin parmi ces 14 jeunes, 7 ont eu recours aux organismes institutionnels - comme l'A.N.P.E. (Agence Nationale Pour l'Emploi) ou les agences d'intérim - ou ont bénéficié dans l'accès à l'emploi d'informations fournies par une personne ne faisant pas partie de leur groupe d'appartenance.

16. Le nom du groupe de jeune de l'association ACORT.

17. Il s'agit des soirées dansantes organisées par le groupe de jeunes de l'association. Les personnes qui travaillent à l'organisation de ces soirées le font bénévolement. De l'autre côté, aux jeunes qui souhaitent participer à ces soirées est réclamée une certaine somme d'argent. Les bénéfices ainsi obtenus sont utilisés pour faire fonctionner les autres activités du groupe de jeunes.

18. Il s'agit d'une émission de radio de trois heures hebdomadaires. Son champ de diffusion est la région parisienne, mais le site Internet de la station permet de toucher un espace beaucoup plus large. Par ailleurs, l'utilisation en alternance de la langue française et de la langue turque, comme le contenu des émissions, montre que celles-ci s'adressent essentiellement aux "jeunes turcs ». Cependant, au vu des appels reçus lors des émissions, nous pouvons conclure que le public touché dépasse largement celui des jeunes.

19. Il s'agit d'une branche de l'architecture qui comprend principalement la conception et réalisation de stands, d'espaces événementiels et de supports de présentation pour les produits commercialisés.

20. Rappelons le, les migrants originaires de Turquie résidant dans la région parisienne se situent au plus bas de la hiérarchie sociale. Ainsi, parmi les 93009 migrants, 30029 sont des ouvriers 
qualifiés, 32489 des ouvriers non qualifiés, 4893 sont dans la catégorie socioprofessionnelle d'« artisans » et 4266 occupent un emploi de "personnel des services directs aux particuliers ». Tableaux thématiques : population immigrée, population étrangère, Paris, INSEE, 2002, pp. 68-69, cité in Verda IRTIŞ -DABBAGH (2003).

\section{AUTEUR}

\section{CEREN ÖZTÜRK}

Sociologue, Doctorante URMIS, CNRS-Paris 7, ceren_ozturk@yahoo.fr 